\title{
Modulation of Tumor Cell Metabolism by Laser Photochemotherapy with Cisplatin or Zoledronic Acid In Vitro
}

\author{
PAUL GÜNTHER BAPTIST HEYMANN ${ }^{1}$, KATHARINA SABINE ELISABETH HENKENIUS ${ }^{2}$, THOMAS ZIEBART ${ }^{1}$, \\ ANDREAS BRAUN ${ }^{3}$, KLARA HIRTHAMMER $^{1}$, FRANK HALLING ${ }^{4}$, ANDREAS NEFF $^{1}$ and ROBERT MANDIC ${ }^{5}$ \\ Departments of ${ }^{1}$ Oral and Maxillofacial Surgery, ${ }^{2}$ Hematology, Oncology and Immunology, \\ ${ }^{5}$ Otorhinolaryngology, Philipps University of Marburg, \\ University Hospital Giessen and Marburg, Campus Marburg, Marburg, Germany; \\ ${ }^{3}$ Department of Operative Dentistry and Endodontology, Philipps University of Marburg, Marburg, Germany; \\ ${ }^{4}$ Medical Practice, Fulda, Germany
}

\begin{abstract}
Background/Aim: Laser photochemotherapy is a new approach in cancer treatment using low-level laser therapy (LLLT) to enhance the effect of chemotherapy. Materials and Methods: In order to evaluate the effect of LLLT on tumor cells, HeLa cells were treated with cisplatin or zoledronic acid (ZA) followed by LLLT. Cell viability was evaluated with 2,3-bis-(2-methoxy-4-nitro-5-sulfophenyl)- $2 \mathrm{H}$ tetrazolium-5-carboxanilide assay. Oxidative phosphorylation and glycolysis were measured using extracellular flux analysis. Immunocytochemistry of heat-shock protein 70 (HSP70) and western blot analysis were performed. Results: LLLT alone increased viability and was associated with lower oxidative phosphorylation but higher glycolysis rates. Cisplatin and ZA alone lowered cell viability, glycolysis and oxidative phosphorylation. This effect was significantly enhanced in conjunction with LLLT and was accompanied by reduced oxidative phosphorylation and collapse of glycolysis. Conclusion: Our observations indicate that LLLT may raise the cytotoxicity of cisplatin and ZA by modulating cellular metabolism, pointing to a possible application in cancer treatment.
\end{abstract}

This article is freely accessible online.

Correspondence to: Dr. Dr. Paul Günther Baptist Heymann, Department: Philipps-Universität Marburg, Clinic for Oral \& Maxillofacial Surgery, Baldingerstrasse, D-35037 Marburg, Germany. Tel: +49 64215869905, Fax: +49 64215868990, e-mail: heymann.paul@gmail.com

Key Words: Cisplatin, zoledronic acid, glycolysis, mitochondria, metabolic escape.
Current knowledge in the area of cellular energy metabolism is largely based on experiments with differentiated non-malignant tissues. In the presence of oxygen, these cells primarily metabolize glucose through mitochondrial oxidative phosphorylation. Under anaerobic conditions, the same cells produce high amounts of lactate (anaerobic glycolysis) (1). In contrast, tumor cells have a significantly different metabolism. Otto Warburg was the first to recognize that cancer cells exhibit high rates of glycolysis even under aerobic conditions. This phenomenon is known as the Warburg effect or aerobic glycolysis (2). A large body of evidence indicates that increased glycolysis in conjunction with reduced mitochondrial respiration are the essential changes in metabolic reprogramming $(1,3,4)$.

Today, chemotherapy plays a central role in cancer treatment. Zoledronic acid (ZA) and cisplatin are widely used chemotherapeutic agents. The bisphosphonate ZA is used for the treatment of several bone-related diseases, such as Paget's disease, postmenopausal osteoporosis and in patients with bone metastases (5). Additionally, several in vitro studies suggested that ZA is able to induce apoptosis and to inhibit cell proliferation $(6,7)$. Cisplatin is one of the most effective agents in the therapy of many solid tumor entities, such as lung, head and neck, ovarian, cervical and testicular cancer (8). Cytotoxicity of cisplatin can be increased by different laser-mediated applications. This was reported for laser thermal therapy (9), low-level laser therapy (LLLT) (10) and in combination with an additional photosensitizer for photodynamic therapy (11-18). Evaluating the concept of laser photochemotherapy, consisting of chemotherapeutic agents activated by LLLT, we showed that LLLT enhances the cytotoxicity of ZA and cisplatin towards primary human fibroblasts and head and neck squamous cell carcinoma (HNSCC) cells (10), as well as normal and malignant bone cells in vitro (19). However, the effect of laser-mediated 
chemotherapy on cellular viability and metabolism of cancer cells is incompletely understood. Here, we investigated the impact of cisplatin and ZA on viability and metabolism of human cervical cancer cells, known to be bone-metastatic $(20,21)$, as well as sensitive to both these chemotherapeutic agents (22). Furthermore, we evaluated the influence of LLLT on these drugs, testing whether laser irradiation is able to raise the cytotoxicity of cisplatin and ZA by altering cellular metabolism in these cells.

\section{Materials and Methods}

Culturing and treatment of HeLa cells with laser photochemotherapy. HeLa cells were kindly provided by B. Matija Peterlin (University of California, San Francisco, CA, USA). Genotyping was performed by a commercial provider (DSMZ GmbH, Braunschweig, Germany) using nonaplex polymerase chain reaction, confirming the cell line to be identical with HeLa cells. Frozen stocks of genotyped HeLa cells were used for the studies presented here. Cells were incubated at $5 \%$ $\mathrm{CO}_{2}\left(37^{\circ} \mathrm{C}\right)$ in Dulbecco's modified Eagle's medium supplemented with $10 \%$ fetal calf serum. Cells were plated at a density of $3 \times 10^{4}$ cells/well in 96-well polystyrene tissue-culture plates. After $24 \mathrm{~h}$ of cultivation, either $6.5,13$ or $26 \mu \mathrm{M}$ cis-diamminedichloroplatinum(II) (Sigma-Aldrich Laborchemikalien $\mathrm{GmbH}$, Seelze, Germany) or 50, 100 and $200 \mu \mathrm{M}$ ZA (Zometa ${ }^{\circledR}$; Novartis AG, Basel, Switzerland, stock: $4 \mathrm{mg} / 5 \mathrm{ml}$ ) was added according to published half maximal inhibitory concentration $\left(\mathrm{IC}_{50}\right)$ values for cisplatin (23) and ZA (24). Wells without agent served as controls. Twenty-four hours after incubation, cells were exposed to LLLT $(\lambda=670 \mathrm{~nm}, 120 \mathrm{~s}, 100$ $\mathrm{mW} / \mathrm{cm}^{2}$ at continuous wave mode with no additional filters) emitted from a non-thermal diode laser (Helbo, Bredent, Walldorf, Germany). For this, the designated laser tip for surface irradiation with a distance of $8 \mathrm{~mm}$ to the cell layer was used. The integrated spacer provided a constant tip-to-sample spacing and thereby guaranteed constant power density for laser treatment. Non-irradiated wells were used as normal controls. The whole experimental setup was carried out under sterile conditions in a vertical laminar flow cabinet. In order to avoid potential scattered irradiation, teflon separators were sited among the wells and the 96-well plate was placed on a black surface during LLLT. The lid of the plate was removed and all wells except for the one to be irradiated were covered. The hand piece of the laser was positioned with the plate by a fixed holder, which was moved from well to well for each irradiation step. After $24 \mathrm{~h}$, a second round of irradiation was performed in the same manner as described above. Three hours after the last irradiation, cell viability was analyzed with sodium 3'-[1-(phenylaminocarbonyl)-3,4tetrazolium]-bis (4-methoxy6-nitro) benzene sulfonic acid hydrate (XTT) assay. For metabolic analyses, the oxygen consumption rate (OCR) and extracellular acidification rate (ECAR) were measured by an Extracellular Flux Analyzer (Seahorse Bioscience, North Billerica, MA, USA). Glycolysis, glycolytic reserve and the Warburg effect were calculated from these data (Figure 1). The influence of treatment on HeLa cells was studied in at least three independent triplicate experiments.

In order to evaluate a thermal impact of LLLT, on tumor cells, the expression of heat-shock protein 70 (HSP70) was evaluated by immunocytochemistry and western blot analysis. To induce HSP70 expression (positive control), cells were incubated for $6 \mathrm{~h}$ at $42.5^{\circ} \mathrm{C}$ in six-well cell-culture dishes as described elsewhere (25). In a second group, cells were laser irradiated for $15 \mathrm{~min}$ and in a third group cells underwent heat shock for $15 \mathrm{~min}$ at $42.5^{\circ} \mathrm{C}$ corresponding to the duration of the laser treatment.

Metabolic assays. OCR and ECAR of HeLa cells were measured in real-time using a Seahorse XFe96 Extracellular Flux Analyzer (Seahorse Bioscience) using the XF Glycolytic Stress Test Kit as recommended by the manufacturer. If not stated differently, cells were plated at a density of $3 \times 10^{4} /$ well (96 wells) and incubated overnight at $37^{\circ} \mathrm{C}$ with $5 \% \mathrm{CO}_{2}$.

In order to measure glycolysis, the maximal ECAR after glucose injection was subtracted from the ECAR prior to glucose injection (Figure 1A). Glycolysis reserve was calculated using the measurement after oligomycin injection subtracted from the ECAR after glucose addition. To determine oxidative phosphorylation, the OCR was measured at baseline (Figure 1B). Values were BCA (Pierce BCA Protein Assay Kit; Thermo Fisher Scientific) normalized prior to calculation of all parameters. According to the manufacturer's protocol, in order to estimate the Warburg effect, non-normalized samples were used by dividing ECAR after glucose injection by OCR values at baseline. After performing the measurement, the cell plate reading was normalized using BCA supplied by the manufacturer's as recommended. Normalization of XF96 raw data was achieved by dividing the values for each well by its respective protein concentration to correct for potential proliferative effects during incubation and loss of cell material during cell preparation.

The XTT cell proliferation assay kit was used according to the manufacturer's protocol (Roche Diagnostics GmbH, Mannheim, Germany). Formation of orange formazan salt by metabolic active cells was measured $3 \mathrm{~h}$ after incubation with the yellow tetrazolium salt [450 nm and $630 \mathrm{~nm}$ (reference), DTX880 Multimode Detector; Beckman Coulter, Inc., Mississauga Ontario, Canada].

Immunocytochemistry. HeLa cells were grown under standard cell culture conditions on coverslips in six-well cell-culture dishes. Cells were treated with LLLT or high temperature $\left(42.5^{\circ} \mathrm{C}\right)$ as described above. Immunocytochemistry was performed as described earlier using anti-HSP70 (1:250, mouse monoclonal, ab5349; Abcam; Cambridge, UK) (26). Secondary anti-mouse (sc-2010) fluorescein isothiocyanate (FITC)-coupled antibodies were from Santa Cruz Biotechnology, Inc (Heidelberg, Germany).

Sodium dodecyl sulfate polyacrylamide gel electrophoresis (SDS PAGE) and western blot analysis. SDS-PAGE and western blot analysis were performed as previously described (26). In order to detect bands of interest, primary antibodies directed against HSP70 (Abcam ab5439, 1:1,000), proliferating cell nuclear antigen (PCNA) (mouse monoclonal, sc-56, 1:500; Santa Cruz Biotechnology, Inc.) or $\beta$-actin (mouse monoclonal, A5441, 1:2,000; Sigma-Aldrich ${ }^{\circledR}$, St. Louis, MO, USA) were added to the nitrocellulose membranes followed by incubation $(1: 2,000)$ with an appropriate secondary goat anti-mouse (sc-2005) horseradish peroxidase-coupled antibody (Santa Cruz Biotechnology, Inc.). Bands were visualized with the enhanced chemiluminescence method (Amersham Biosciences, Buckinghamshire, UK) on X-ray film (Agfa, Cologne, Germany). Western blots were carried out in triplicate. All bands were quantified with the program ImageJ 1.48v (27) and were normalized to $\beta$-actin prior to statistical analysis.

Statistical analysis. For statistical analysis, IBM SPSS Statistics Version 22.0 (IBM Corp., Armonk, NY, USA) was used. Data are 


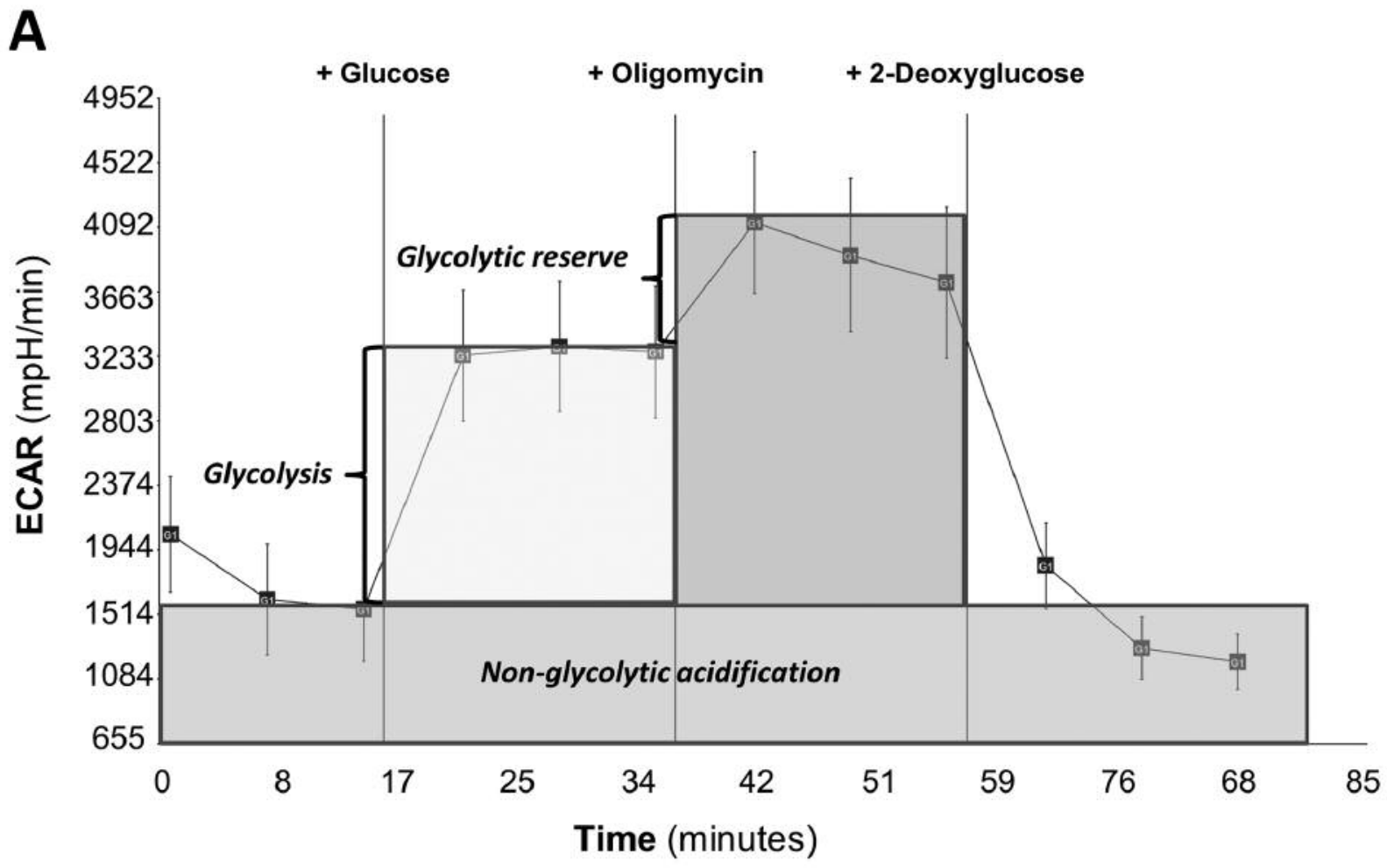

B

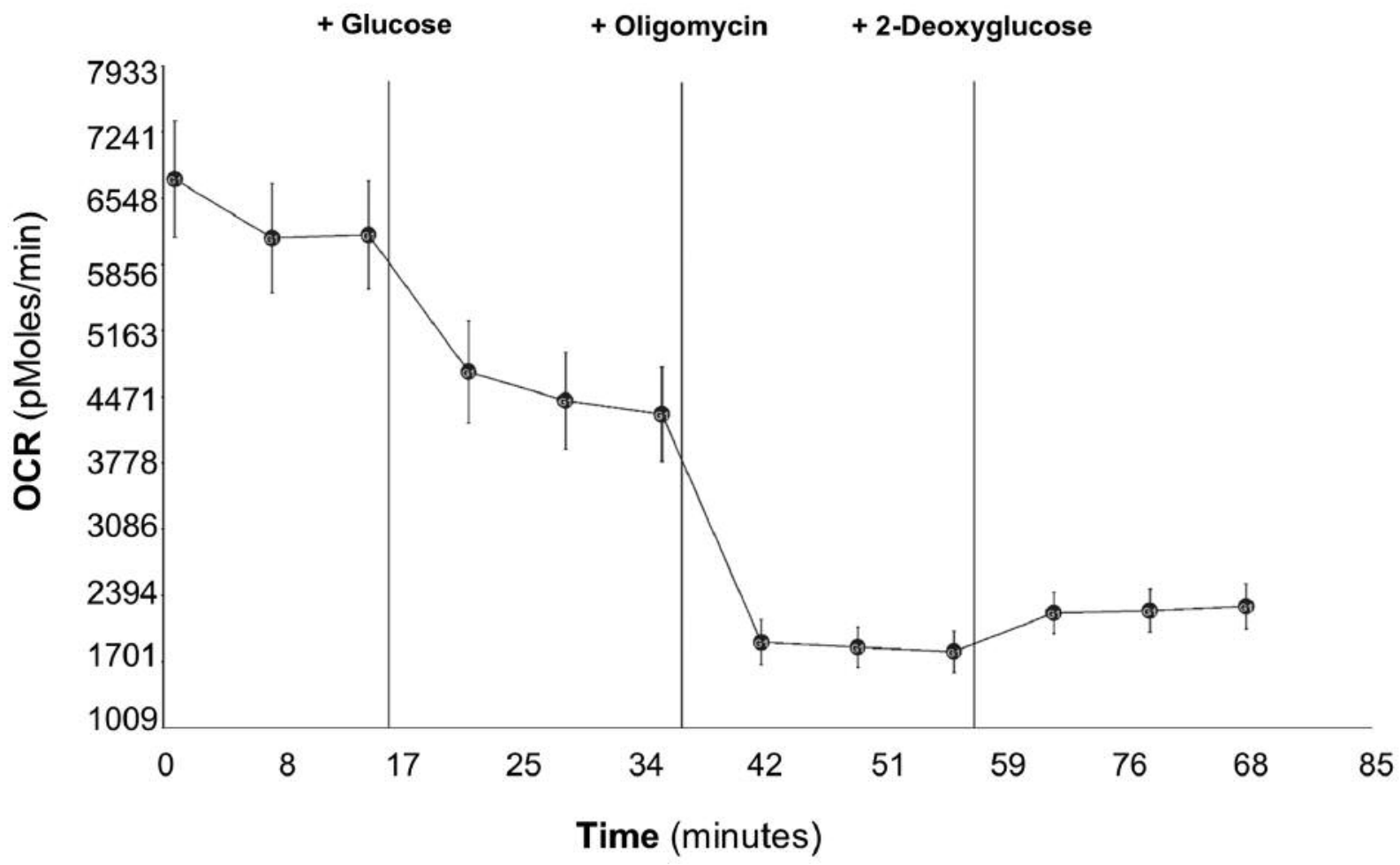

Figure 1. Measuring extracellular acidification rate (ECAR) for glycolytic flux (A) and oxygen consumption rate (OCR) for oxidative phosphorylation (B) in HeLa cells. Metabolic response of HeLa cells to glucose $(10 \mathrm{mM})$, oligomycin $(5 \mu \mathrm{M})$ and 2-deoxyglucose $(100 \mathrm{mM})$. Data are the mean \pm SEM, $n=6$. 


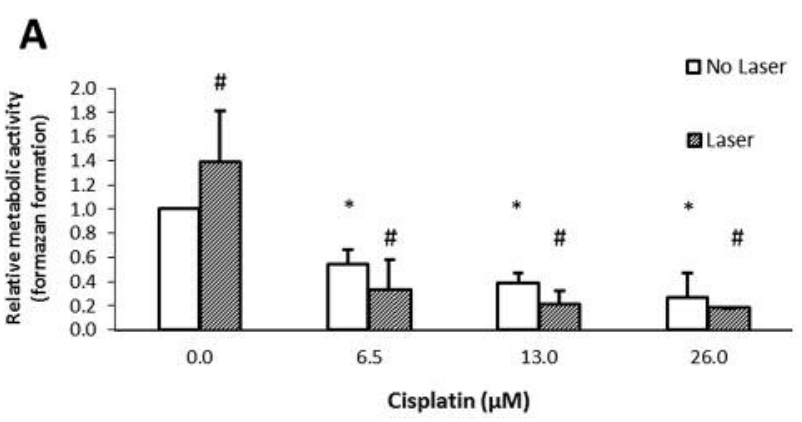

B

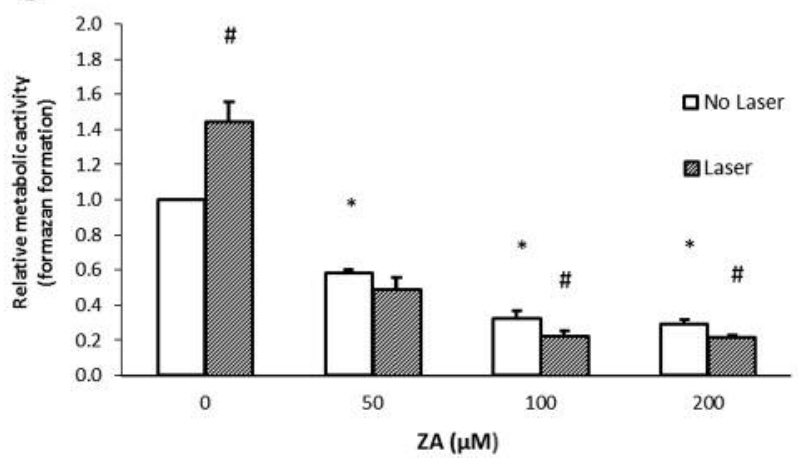

Figure 2. Evaluation of the relative metabolic activity in HeLa cells after incubation with cisplatin (A), and zoledronic acid (ZA) (B) with and without low-level laser therapy (LLLT). Significantly different at ${ }^{*} p<0.05$ (chemotherapy vs. untreated), ${ }^{\#} p<0.05$ (LLLT vs. untreated), data are presented as the mean of 3 experiments $\pm S E M$.

shown as the mean and standard error of the mean (SEM), with at least three independent replicates used for each data point. Normal distribution was assessed with the Shapiro-Wilk test. As data were not normally distributed, differences between the groups were analyzed with a non-parametric test (Kruskal-Wallis) and MannWhitney pairwise comparisons $(p<0.05)$. Differences were considered as statistically significant at $p<0.05$. For comparison of western blot bands after quantification with ImageJ, values were calculated using the one-sided paired t-test.

\section{Results}

Effect of cisplatin and ZA on HeLa cell viability. Cisplatin significantly lowered viability of HeLa cells at all concentrations used $(6.5,13$ and $26 \mu \mathrm{M})$ in comparison to the untreated controls $(p=0.001)$ (Figure 2A). Similar results were obtained for ZA, cells treated with 50,100 or $200 \mu \mathrm{M}$ ZA demonstrated a significantly reduced cellular viability $(p=0.001)$ (Figure 2B).

Laser therapy. Interestingly, LLLT alone significantly increased the viability of HeLa cells compared to nonirradiated control cells $(p=0.029)(0 \mu \mathrm{M}$; Figure $2 \mathrm{~A}$ and $\mathrm{B})$. LLLT in conjunction with cisplatin treatment impaired cell viability at every cisplatin concentration tested compared with the control group (cisplatin only) $(6.5,13$ and $26 \mu \mathrm{M}$ : $p=0.008, p=0.039$ and $p=0.001$, respectively). Similar effects were observed after ZA exposure. Here, LLLT significantly reduced cell viability at the two highest concentrations of 100 and $200 \mu \mathrm{M}$ of ZA $(p=0.039$ and $p<0.001)$ (Figure 2B). Although showing a reduction, no significant difference in viability was found after LLLT in the presence of $50 \mu \mathrm{M}$ ZA $(p=0.345)$.

Effect of cisplatin and ZA on HeLa cell metabolism. Extracellular flux analysis was performed on HeLa cells exposed to $13 \mu \mathrm{M}$ cisplatin (Figure 3) or $100 \mu \mathrm{M}$ ZA (Figure 4). Cisplatin significantly inhibited oxidative phosphorylation $(p<0.001)$ (Figure 3A), glycolysis $(p=0.00539)$ (Figure 3B) and glycolytic reserve $(p<0.001)$ (Figure $3 \mathrm{C})$. The Warburg effect was significantly enhanced in comparison to untreated control groups $(p<0.001)$ (Figure 3D).

Similar results were seen after incubation with $100 \mu \mathrm{M}$ ZA (Figure 4). Oxidative phosphorylation $(p<0.001)$ (Figure $4 \mathrm{~A})$, glycolysis $(p=0.015)$ (Figure $4 \mathrm{~B})$ and glycolytic reserve $(p=0.009)$ (Figure 4C) were significantly impaired, with a concomitant increase in the Warburg effect $(p=0.039)$ (Figure 4D).

Laser therapy. LLLT alone significantly increased glycolysis $(p=0.0026)$ (Figure 3B and 4B) and reduced glycolytic reserve $(p=0.0025)$ (Figure 3C and 4C). Although oxidative phosphorylation was diminished $(p=0.0039)$ (Figure $3 \mathrm{~A}$ and $4 \mathrm{~A}$ ), the Warburg effect, defined as the ratio between ECAR and OCR, revealed significantly higher values in comparison to non-irradiated control cells $(p<0.001)$ (Figure 3D and 4D).

In the presence of $13 \mu \mathrm{M}$ cisplatin, supplemental LLLT significantly reduced oxidative phosphorylation $(p<0.029)$ (Figure 3A), glycolysis ( $p=0.042$ ) (Figure 3B) and glycolytic reserve $(p=0.037)$ (Figure $3 \mathrm{C})$, whereas the Warburg effect was elevated in comparison to cisplatin treatment alone $(p<0.001)$ (Figure 3D). In the presence of $100 \mu \mathrm{M} \mathrm{ZA}$, additional LLLT generated a significant reduction of oxidative phosphorylation ( $p=0.0019)$ (Figure 4A), glycolysis $(p=0.041)$ (Figure 4B) and glycolytic reserve $(p=0.028)$ (Figure 4C), whereas the Warburg effect was enhanced in comparison to ZA treatment alone $(p<0.001)$ (Figure 4D).

Influence of laser-mediated heat. To evaluate the presence of thermal effects due to LLLT, we investigated the expression of HSP70, as well as that of the cellular proliferation marker PCNA in HeLa cells. Immunocytochemistry showed HeLa cells without heat exposure exhibited basal expression levels of HSP70, which appeared markedly induced after heat exposure $\left(42.5^{\circ} \mathrm{C}\right)$ for $6 \mathrm{~h}$ but not after short $(15 \mathrm{~min})$ heat $\left(42.5^{\circ} \mathrm{C}\right.$ ) exposure or LLLT for $15 \mathrm{~min}$ (Figure 5A). Western blot analysis supported these observations (Figure 5B). Here, 

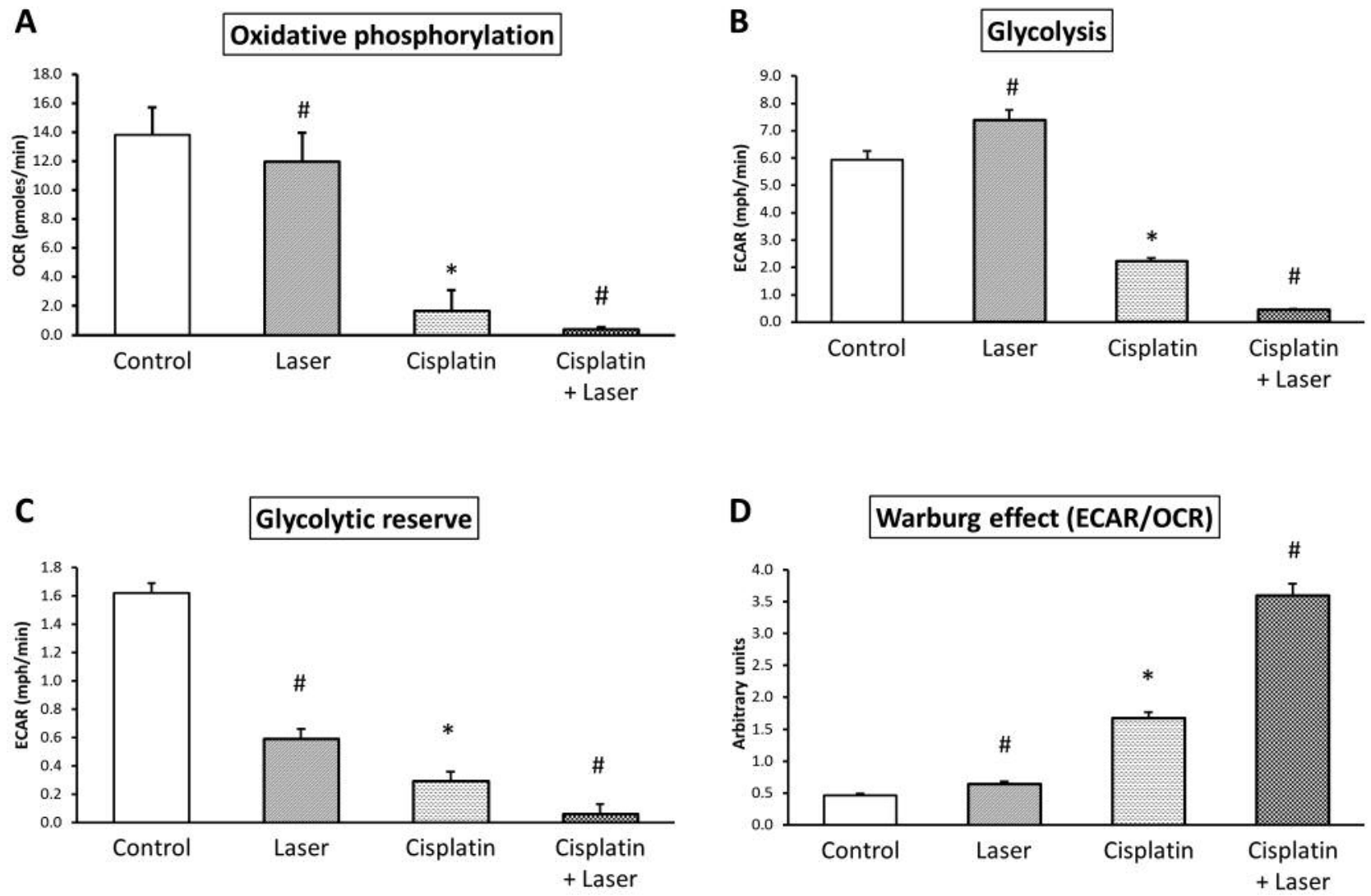

Figure 3. Multiparameter metabolic analysis of measuring oxygen consumption rate (OCR) for oxidative phosphorylation (A) and extracellular acidification rate $(E C A R)$ for glycolysis $(B)$, glycolytic reserve $(C)$ and for the Warburg effect $(D)$ in the presence of $13 \mu M$ cisplatin $(C D D P)$ with and without low-level laser therapy (LLLT). Significantly different at ${ }^{*} p<0.05$ (chemotherapy vs. untreated), ${ }^{*} p<0.05$ (LLLT vs. untreated). Data are means \pm SEM.

a reduction of PCNA, reflecting the proliferative state of the cell, was also seen (Figure 5B). Induction of HSP70 and reduction of PCNA expression in long-term $(6 \mathrm{~h})$ heat-treated $\left(42.5^{\circ} \mathrm{C}\right)$ cells was statistically significant $(p=0.0305$ and $p=0.0254$ ) (Figure 5C).

Influence of treatment on PCNA expression. Treatment of HeLa cells with cisplatin or a combination of cisplatin and LLLT led to an average reduction (not significant) of PCNA protein expression (Figure 6A). Treatment of cells with ZA or a combination of cisplatin and LLLT significantly lowered the PCNA expression level ( $p=0.035$ and $p=0.0178$, respectively) (Figure $6 \mathrm{~B}$ ).

\section{Discussion}

Cancer cell metabolism is characterized by high rates of glycolysis, which makes tumor cells highly dependent on glucose for survival (28). The potential of changing tumor cell metabolism for therapeutic benefit using LLLT was investigated, based on the assumption that manipulation of cellular metabolism by laser light may chemosensitize cancer cells.

Cisplatin and ZA are widely used chemotherapeutic agents in modern oncology. Cisplatin is typically deployed in the treatment of solid tumors (8). Clinical indications for use of the bisphosphonate ZA are inhibition of bone loss associated with several bone diseases, as well as tumor metastasis to bone (5). In which way the cellular metabolism of bone metastatic human cervical cancer is influenced by LLLT alone or in combination with cisplatin or ZA is incompletely understood.

Cisplatin lowered the viability of HeLa cells at all tested concentrations. Such a dose-dependent cytotoxicity is well established $(23,29)$. Similar results were also reported for ZA by other study groups (24).

Focusing on tumor cell metabolism, both chemotherapeutic agents reduced oxidative phosphorylation, glycolysis and glycolytic reserve, while enhancing the Warburg effect. The described effects were more distinct for cisplatin than for ZA. There is growing evidence that cisplatin, as well as its action 

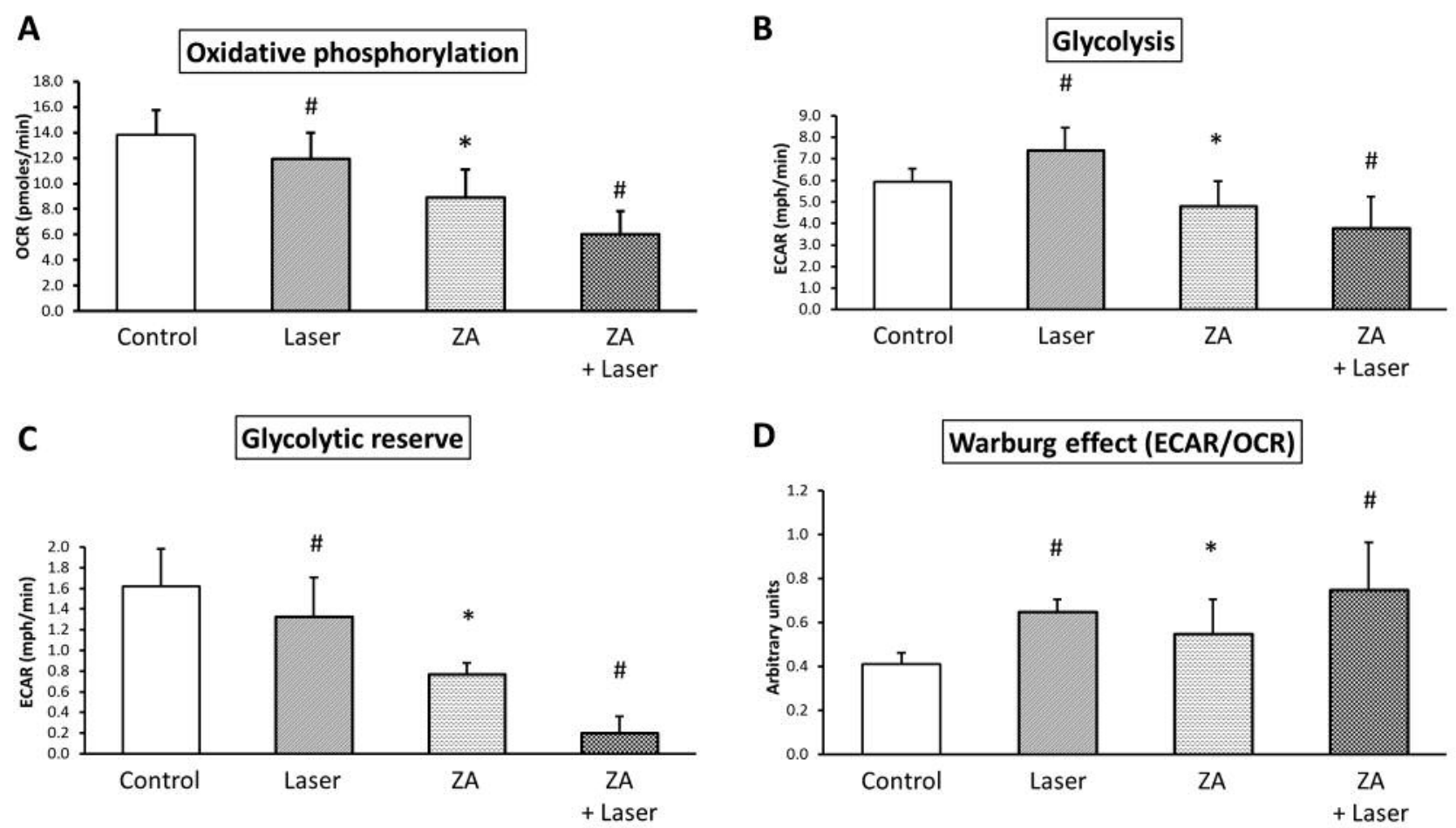

Figure 4. Multiparameter metabolic analysis of oxygen consumption rate (OCR) for oxidative phosphorylation (A) and extracellular acidification rate $(E C A R)$ for glycolysis $(B)$, glycolytic reserve $(C)$ and for the Warburg effect $(D)$ in the presence of $100 \mu M$ zoledronic acid $(Z A)$ with and without low-level laser therapy (LLLT). Significantly different at ${ }^{*} p<0.05$ (chemotherapy vs. untreated), ${ }^{\#} p<0.05$ (LLLT vs. untreated). Data are means $\pm S E M$.

on genomic DNA, has anti-metabolic activities resulting in reduced glycolysis and oxidative phosphorylation, as was seen in the present study. Wang et al. reported for the human uterine cervical cancer cell line $\mathrm{SiHa}$ that cisplatin reduced glycolysis and suppressed glycolysis-related protein expression, including that of glucose transporter 1 and 4 (30). Furthermore, mitochondrial accumulation of cisplatin was reported to damage mitochondrial structure and function (31). This could be one possible explanation for the impaired cellular respiration observed in our study for HeLa cells and by Alborzinia et al. for the colon carcinoma cells HT-29 and HCT-116 (32).

The influence of bisphosphonates such as ZA on the metabolism of tumor cells remains unclear. Kopecka et al. reported the decrease of glycolysis and the inhibition of oxidative phosphorylation, as observed in our study, for multidrug-resistant cancer cells (33). The distinct molecular effects of bisphosphonates on mitochondria are yet to be clarified. It was suggested that their observed pro-apoptotic effect on tumor cells, resulting in reduced invasion, adhesion, proliferation, and angiogenesis, could directly be induced via a mitochondria-dependent pathway (34), possibly being associated with indirect inhibition of protein isoprenylation or direct inhibition of the mitochondrial enzyme adenine nucleotide translocase through the ATP analog 1-adenosin-5'-yl ester 3-(3-methylbut-3-enyl) ester $(35,36)$.

LLLT of ZA- or cisplatin-treated HeLa cells reduced cell viability even more than did the single agents. Lower levels of oxidative phosphorylation, glycolysis and glycolytic capacity accompanied this treatment, whereas the Warburg effect appeared to be induced. The increasing cellular effect on cisplatin-induced toxicity after additional irradiation is in accordance with findings of other research groups using different laser-based technologies.

Photodynamic therapy, in which a photosensitizer is activated by laser light of a specific wavelength, enhanced the anticancer effect of cisplatin as reported for colon cancer cells in vitro and in vivo by Ge and co-workers (11), as well as for squamous cell carcinoma in vivo by Uehara et al. (37). Further in vivo research demonstrated that the antitumor effect of a local cisplatin application can be boosted by laser thermal therapy (9) or by interstitial laser therapy (38).

Taken together, LLLT was able to raise the cytotoxicity of the applied drug at nearly every concentration tested, which is known as laser photochemotherapy (39). There is evidence 

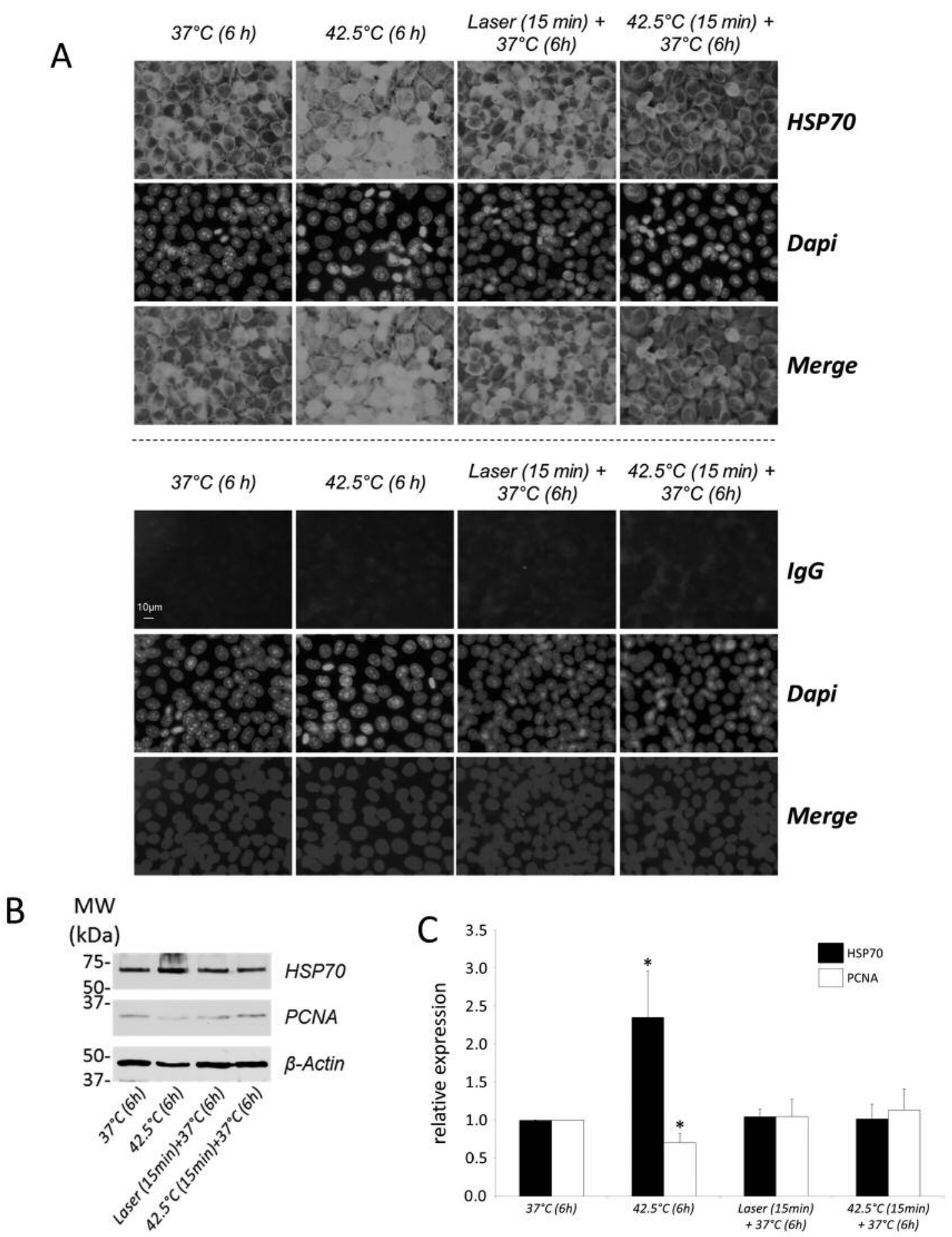

Figure 5. Laser exposure of cultured HeLa cells does not induce expression of heat-shock protein 70 (HSP70). A: Basal expression of HSP70 was detected in non-stressed $\left(37^{\circ} \mathrm{C}, 6 \mathrm{~h}\right)$ as well as all treated cells by immunocytochemistry (upper panel). Only long (6 h) exposure to $42.5^{\circ} \mathrm{C}$ visibly induced $\mathrm{HSP} 70$ expression in HeLa cells, whereas treating cells for 15 min with laser (typical therapeutic duration) or at $42.5^{\circ} \mathrm{C}$ with subsequent incubation for $6 \mathrm{~h}$ at $37^{\circ} \mathrm{C}$ did not appear to affect HSP70 expression levels. The lower panel shows control staining of HeLa cells using normal IgG to document the specificity of the HSP70 antibody. Note that all HSP70 and IgG images [fluorescein isothiocyanate channel (FITC)] were photographed at identical microscope settings (exposure times) to make images comparable with each other. The size bar shown in the upper left photograph of the lower panel is representative of all images. B: Western blot analysis confirms the observation made from (A) that only long (6 h) exposure at $42.5^{\circ} \mathrm{C}$ but not short (15 min) laser or high temperature $\left(42.5^{\circ} \mathrm{C}\right)$ treatment was capable of significantly inducing HSP70 expression over basal levels. Similarly, proliferating cell nuclear antigen (PCNA) expression, as an indicator of cellular proliferation, was significantly reduced only in cells incubated for $6 \mathrm{~h}$ at $42.5^{\circ} \mathrm{C}$. Bands were quantified as described in the Materials and Methods section. Data are means \pm SEM (n=3). *Significantly different at $p<0.05 .4$ ',6-diamidino-2-phenylindole (Dapi). 
A
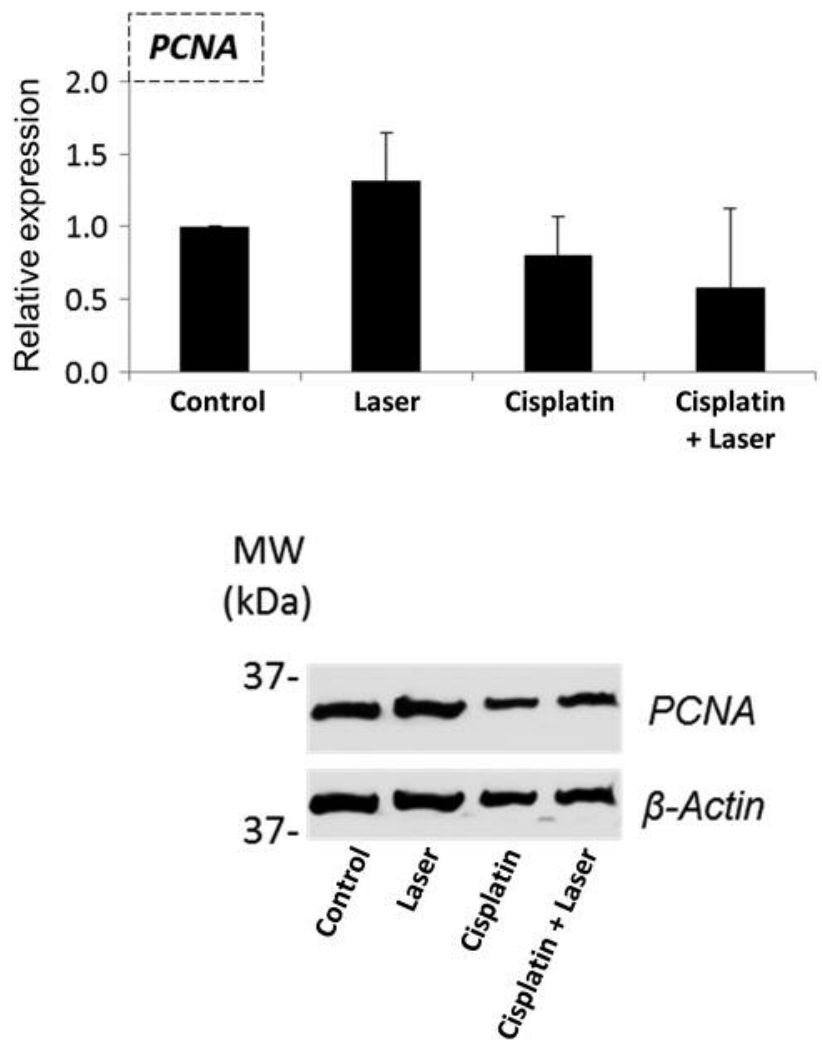

B

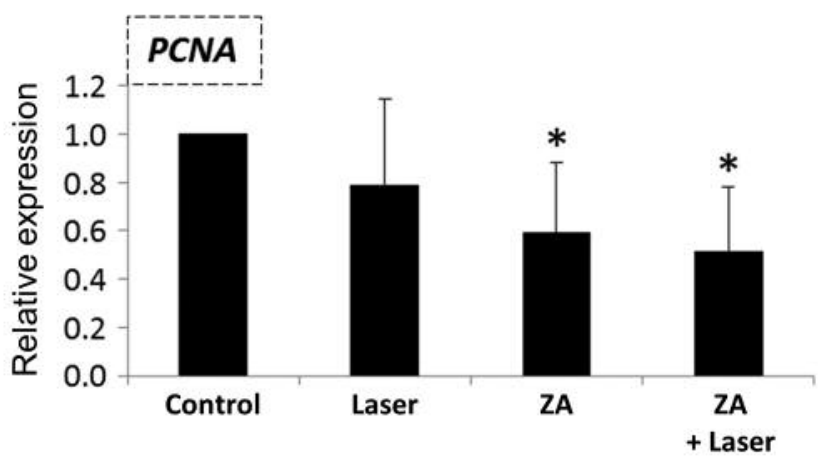

Figure 6. Treatment of HeLa cells with laser or in combination with cisplatin or zoledronic acid (ZA) affects expression of proliferating cell nuclear antigen (PCNA). A: Western blot analysis revealed an average reduction (not statistically significant) in the expression of the proliferation marker PCNA in HeLa cells treated with CDDP or a combination of laser and CDDP $(n=3, t$-test $)$. B: Similarly, PCNA expression was also reduced $(p<0.05)$ in HeLa cells treated with ZA or a combination of laser and ZA $(n=4, t$-test $)$. Bands were quantified as described in the Materials and Methods section. *Significantly different at $p<0.05$.

that chemotherapeutic agents such as cisplatin are potential candidates for light or heat activation in tumor cells (40).

Furthermore, it has been demonstrated that tumor cells are able to switch between glycolysis and mitochondrial metabolism (oxidative phosphorylation) depending on environmental conditions $(41,42)$. Since cisplatin and ZA both have anti-glycolytic activities, glycolysis of HeLa cells will be disturbed after exposure of cells to either of the two compounds. Compensation of this anti-glycolytic activity can result in a higher mitochondrial respiration rate, in the sense of metabolic escape (43). As single LLLT without the presence of cytostatic drugs was able to lower mitochondrial respiration, LLLT in the context of laser photochemotherapy could help to disable such a metabolic escape of glycolysis-impaired cells by inhibiting mitochondrial function (Figure 7). This may also explain the observations that LLLT increased cytotoxicity of cisplatin or ZA by additional reduction of cellular respiration, thereby avoiding bioenergetic adaptation, resulting in cell growth inhibition. However, the impact of laser irradiation on HeLa cells appears to be ambivalent. Without chemotherapy, LLLT increased viability and metabolism of HeLa cells, which has also been reported in recent literature for other cell lines (44). Interestingly, LLLT was able to enhance cytotoxicity of chemotherapeutic agents, possibly by direct impact on glycolysis and mitochondrial function.

As a proof of principle, the present study evaluated the effect of laser photochemotherapy in conjunction with cisplatin or ZA on the human cervical cancer cell line HeLa. Cervical cancer is one of the most frequent malignancies affecting women, with a 5-year survival rate of only $16.5 \%$ for patients with metastatic disease (45). There is growing evidence that cisplatin-based chemotherapy has a positive impact on survival (46). As bone metastases are present in up to $22.9 \%$ of patients (21), bisphosphonates such as ZA are clinically effective (47), even though no positive effects on survival rates were observed (48). 
Glycolysis

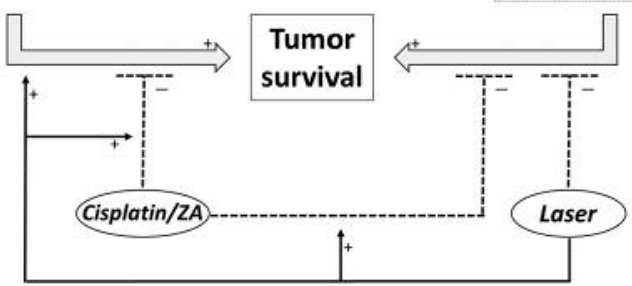

Oxidative phosphorylation

Figure 7. Scheme depicting the possible influence of laser photochemotherapy on tumor cell metabolism. Preventing metabolic escape of glycolysis (-) arrested HeLa cells due to low-level laser therapy (LLLT)-mediated inhibition of mitochondrial function.

In a clinical setting, laser photochemotherapy comprising cisplatin or ZA together with additional LLLT could help to improve treatment of locally advanced cancer. Another therapeutic concept, e.g. intraoperative brachytherapy after neo-adjuvant chemotherapy with cisplatin, could be used to kill remaining cancer cells or control bone metastases with the addition of ZA. The observation of anti-metabolic properties of cisplatin and ZA in a cell line sensitive to both chemotherapeutic compounds will also be interesting to test in other types of solid tumors such as head and neck cancer.

In conclusion, LLLT can modulate cell viability and metabolism of human cervical cancer cells. In combination with cisplatin and ZA, LLLT increases cellular toxicity, which might be explained by additional LLLT preventing metabolic mitochondrial escape of tumor cells in the presence of cytostatic drugs with anti-glycolytic effects.

The presented approach could be of interest in the evaluation of new therapeutic strategies against locally advanced tumors or when bone metastases are present.

\section{Conflicts of Interest}

The Authors report no conflicts of interest in regard to this study.

\section{Acknowledgements}

Instrumental and technical support during metabolic analysis with the Seahorse XF96 Extracellular Flux Analyzer at the Core Facilities of the Faculty of Medicine (Marburg, Germany), located at the Center for Tumor Biology and Immunology, by the team of PD Dr. C. Brendel (Department of Hematology and Oncology, Marburg, Germany), was greatly appreciated. Furthermore, the Authors would like to thank Ms. Roswitha Peldszus and Ms. Maria Sadowski (Department of Otorhinolaryngology, Head and Neck Surgery, University Hospital Giessen and Marburg, Campus Marburg, Marburg, Germany) for excellent technical support. The Authors would also thank PD. Dr. M. Bette (Department of Anatomy and Cell Biology, Marburg, Germany) for providing support during microscopical analysis.

\section{References}

1 Vander Heiden MG, Cantley LC and Thompson CB: Understanding the Warburg effect: the metabolic requirements of cell proliferation. Science 324: 1029-1033, 2009.

2 Warburg O: On the origin of cancer cells. Science 123: 309-314, 1956.

3 Bol V, Bol A, Bouzin C, Labar D, Lee JA, Janssens G, Porporato PE, Sonveaux $\mathrm{P}$, Feron $\mathrm{O}$ and Gregoire V: Reprogramming of tumor metabolism by targeting mitochondria improves tumor response to irradiation. Acta Oncol 54: 266274, 2015.

4 Wang M, Keogh A, Treves S, Idle JR and Beyoglu D: The metabolomic profile of gamma-irradiated human hepatoma and muscle cells reveals metabolic changes consistent with the Warburg effect. PeerJ 4: e1624, 2016.

5 Russell RG: Bisphosphonates: from bench to bedside. Ann N Y Acad Sci 1068: 367-401, 2006.

6 Senaratne SG, Pirianov G, Mansi JL, Arnett TR and Colston KW: Bisphosphonates induce apoptosis in human breast cancer cell lines. Br J Cancer 82: 1459-1468, 2000.

7 Fu Q, Cui C, Xuan B, Guo Y, Liu C and Zhang J: Effect of zoledronic acid on cell proliferation and apoptosis of human periodontal fibroblasts. Zhonghua Kou Qiang Yi Xue Za Zhi 50: 667-670, 2015 (in Chinese).

8 Basu A and Krishnamurthy S: Cellular responses to cisplatininduced DNA damage. J Nucleic Acids 2010, 2010.

9 Kanekal S, Joo J, Bublik M, Bababeygy A, Loh C, Castro DJ, Sercarz JA and Paiva MB: Retention of intratumor injections of cisplatinum in murine tumors and the impact on laser thermal therapy for cancer treatment. Eur Arch Otorhinolaryngol 266: 279-284, 2009.

10 Heymann PG, Mandic R, Kammerer PW, Kretschmer F, Saydali A, Neff A and Draenert FG: Laser-enhanced cytotoxicity of zoledronic acid and cisplatin on primary human fibroblasts and head and neck squamous cell carcinoma cell line UM-SCC-3. J Craniomaxillofac Surg 42: 1469-1474, 2014.

11 Ge R, Ahn JC, Shin JI, Bahk CW, He P and Chung PS: An in vitro and in vivo study of combination therapy with Photogem(R)-mediated photodynamic therapy and cisplatin on mouse cancer cells (CT-26). Photomed Laser Surg 29: 155-160, 2011.

12 Ahn JC, Biswas R, Mondal A, Lee YK and Chung PS: Cisplatin enhances the efficacy of 5-aminolevulinic acid mediated photodynamic therapy in human head and neck squamous cell carcinoma. Gen Physiol Biophys 33: 53-62, 2014.

13 Biswas R, Chung PS, Moon JH, Lee SH and Ahn JC: Carboplatin synergistically triggers the efficacy of photodynamic therapy via caspase -3-, -8-, and -12-dependent pathways in human anaplastic thyroid cancer cells. Lasers Med Sci 29: 995$1007,2014$.

14 Lee SJ, Hwang HJ, Shin JI, Ahn JC and Chung PS: Enhancement of cytotoxic effect on human head and neck cancer cells by combination of photodynamic therapy and sulforaphane. Gen Physiol Biophys 34: 13-21, 2015.

15 Theodoraki MN, Lorenz KJ, Schneider J, Thierauf JC, Spagnoli G, Schuler PJ, Hoffmann TK and Laban S: Influence of photodynamic therapy on the expression of cancer/testis antigens in squamous cell carcinoma of the head and neck. Anticancer Res 36: 3973-82, 2016. 
16 Bajgar R, Kolarova H, Bolek L, Binder S, Pizova K and Hanakova A: High oxygen partial pressure increases photodynamic effect on HeLa cell lines in the presence of chloraluminium phthalocyanine. Anticancer Res 34: 4095-4099, 2014.

17 Murakami G, Nanashima A, Nonaka T, Tominaga T, Wakata K, Sumida Y, Akashi H, Okazaki S, Kataoka $\mathrm{H}$ and Nagayasu T: Photodynamic therapy using novel glucose-conjugated chlorin increases apoptosis of cholangiocellular carcinoma in comparison with talaporfin sodium. Anticancer Res 36: 44934501, 2016.

18 Horne TK and Cronje MJ: Cancer Tissue Classification, Associated Therapeutic Implications and PDT as an Alternative. Anticancer Res 37: 2785-2807, 2017.

19 Heymann PG, Ziebart T, Kammerer PW, Mandic R, Saydali A, Braun A, Neff A and Draenert GF: The enhancing effect of a laser photochemotherapy with cisplatin or zolendronic acid in primary human osteoblasts and osteosarcoma cells in vitro. $\mathrm{J}$ Oral Pathol Med 45: 803-809, 2016.

20 Wise-Milestone L, Akens MK, Rosol TJ, Hojjat SP, Grynpas MD and Whyne CM: Evaluating the effects of mixed osteolytic/osteoblastic metastasis on vertebral bone quality in a new rat model. J Orthop Res 30: 817-23, 2012.

$21 \mathrm{Li} \mathrm{H}, \mathrm{Wu} \mathrm{X}$ and Cheng X: Advances in diagnosis and treatment of metastatic cervical cancer. J Gynecol Oncol 27: e43, 2016.

22 Burke M, Atkins A, Kiss A, Akens M, Yee A and Whyne C: The impact of metastasis on the mineral phase of vertebral bone tissue. J Mech Behav Biomed Mater 69: 75-84, 2016.

23 Zhu K, Chen L, Han X and Wang J: Short hairpin RNA targeting Twist1 suppresses cell proliferation and improves chemosensitivity to cisplatin in HeLa human cervical cancer cells. Oncol Rep 27: 1027-1034, 2012.

24 Wang IT, Chou SC and Lin YC: Zoledronic acid induces apoptosis and autophagy in cervical cancer cells. Tumour Biol 35: 11913-11920, 2014.

25 Wagner M, Hermanns I, Bittinger $\mathrm{F}$ and Kirkpatrick CJ: Induction of stress proteins in human endothelial cells by heavy metal ions and heat shock. Am J Physiol 277: L1026-33, 1999.

26 Mandic R, Schamberger CJ, Muller JF, Geyer M, Zhu L, Carey TE, Grenman R, Dunne AA and Werner JA: Reduced cisplatin sensitivity of head and neck squamous cell carcinoma cell lines correlates with mutations affecting the $\mathrm{COOH}$-terminal nuclear localization signal of p53. Clin Cancer Res 11: 6845-6852, 2005.

27 Schneider CA, Rasband WS and Eliceiri KW: NIH Image to ImageJ: 25 years of image analysis. Nat Methods 9: 671-675, 2012.

28 Ngo DC, Ververis K, Tortorella SM and Karagiannis TC: Introduction to the molecular basis of cancer metabolism and the Warburg effect. Mol Biol Rep 42: 819-823, 2015;

29 Ujhelyi Z, Kalantari A, Vecsernyes M, Roka E, Fenyvesi F, Poka R, Kozma B and Bacskay I: The enhanced inhibitory effect of different antitumor agents in self-microemulsifying drug delivery systems on human cervical cancer HeLa cells. Molecules 20 : 13226-13239, 2015.

30 Wang S, Xie J, Li J, Liu F, Wu X and Wang Z: Cisplatin suppresses the growth and proliferation of breast and cervical cancer cell lines by inhibiting integrin beta5-mediated glycolysis. Am J Cancer Res 6: 1108-1117, 2016.
31 Dzamitika S, Salerno M, Pereira-Maia E, Le Moyec L and Garnier-Suillerot A: Preferential energy- and potential-dependent accumulation of cisplatin-gutathione complexes in human cancer cell lines (GLC4 and K562): A likely role of mitochondria. J Bioenerg Biomembr 38: 11-21, 2006.

32 Alborzinia H, Can S, Holenya P, Scholl C, Lederer E, Kitanovic I and Wolfl S: Real-time monitoring of cisplatin-induced cell death. PLoS One 6: e19714, 2011.

33 Kopecka J, Porto S, Lusa S, Gazzano E, Salzano G, Giordano A, Desiderio V, Ghigo D, Caraglia M, De Rosa G and Riganti C: Self-assembling nanoparticles encapsulating zoledronic acid revert multidrug resistance in cancer cells. Oncotarget 6: 3146131478, 2015.

34 Gao X, Jiang B, Zou S, Zhang T, Qi X, Jin L, Ge X, Tang SC, Hua D and Chen W: Zoledronate can promote apoptosis and inhibit the proliferation of colorectal cancer cells. Tumour Biol 36: 5315-5322, 2015.

35 Monkkonen H, Auriola S, Lehenkari P, Kellinsalmi M, Hassinen IE, Vepsalainen J and Monkkonen J: A new endogenous ATP analog (ApppI) inhibits the mitochondrial adenine nucleotide translocase (ANT) and is responsible for the apoptosis induced by nitrogen-containing bisphosphonates. Br J Pharmacol 147: 437-445, 2006.

36 Raikkonen J, Monkkonen H, Auriola S and Monkkonen J: Mevalonate pathway intermediates downregulate zoledronic acid-induced isopentenyl pyrophosphate and ATP analog formation in human breast cancer cells. Biochem Pharmacol 79: 777-783, 2010.

37 Uehara M, Inokuchi $\mathrm{T}$ and Ikeda $\mathrm{H}$ : Enhanced susceptibility of mouse squamous cell carcinoma to photodynamic therapy combined with low-dose administration of cisplatin. J Oral Maxillofac Surg 64: 390-396, 2006.

38 Graeber IP, Eshraghi AA, Paiva MB, Paek WH, Castro DJ, Jovanovic S, Scherer H, Soudant J and Saxton RE: Combined intratumor cisplatinum injection and Nd:YAG laser therapy. Laryngoscope 109: 447-454, 1999.

39 Saxton RE, Paiva MB, Lufkin RB and Castro DJ: Laser photochemotherapy: a less invasive approach for treatment of cancer. Semin Surg Oncol 11: 283-289, 1995.

40 Paiva MB, Joo J, Abrahao M, Ribeiro JC, Cervantes O and Sercarz JA: Update on laser photochemotherapy: an alternative for cancer treatment. Anticancer Agents Med Chem 11: 772-779, 2011.

41 Rossignol R, Gilkerson R, Aggeler R, Yamagata K, Remington SJ and Capaldi RA: Energy substrate modulates mitochondrial structure and oxidative capacity in cancer cells. Cancer Res 64 : 985-993, 2004.

42 Smolkova K, Bellance N, Scandurra F, Genot E, Gnaiger E, Plecita-Hlavata L, Jezek P and Rossignol R: Mitochondrial bioenergetic adaptations of breast cancer cells to aglycemia and hypoxia. J Bioenerg Biomembr 42: 55-67, 2010.

43 Plecita-Hlavata L, Lessard M, Santorova J, Bewersdorf J and Jezek P: Mitochondrial oxidative phosphorylation and energetic status are reflected by morphology of mitochondrial network in INS-1E and HEP-G2 cells viewed by 4Pi microscopy. Biochim Biophys Acta 1777: 834-846, 2008.

44 Ginani F, Soares DM, Barreto MP and Barboza CA: Effect of low-level laser therapy on mesenchymal stem cell proliferation: a systematic review. Lasers Med Sci 30: 21892194, 2015. 
45 Ferlay J, Steliarova-Foucher E, Lortet-Tieulent J, Rosso S, Coebergh JW, Comber H, Forman D and Bray F: Cancer incidence and mortality patterns in Europe: estimates for 40 countries in 2012. Eur J Cancer 49: 1374-1403, 2013.

46 Vermorken JB: The role of chemotherapy in squamous cell carcinoma of the uterine cervix: a review. Int J Gynecol Cancer 3: 129-142, 1993.

47 Tsubamoto H, Inoue K, Ukita Y, Ito Y and Kanazawa R: Longterm remission after multiple bone metastases following cervical cancer: A case report. Gynecol Oncol Case Rep 5: 22-24, 2013.
48 Mackiewicz-Wysocka M, Pankowska M and Wysocki PJ: Progress in the treatment of bone metastases in cancer patients. Expert Opin Investig Drugs 21: 785-795, 2012.

Received November 24, 2017

Revised December 15, 2017

Accepted December 18, 2017 